\title{
eJRIEPS
}

Ejournal de la recherche sur l'intervention en éducation physique et sport

Hors-série $N^{\circ} 2 \mid 2018$

À propos de certaines bases théoriques et pratiques des sports collectifs

\section{Les jeux à effectif réduit : plaisir et apprentissage}

Jean-Francis Gréhaigne

\section{OpenEdition}

Journals

Édition électronique

URL : http://journals.openedition.org/ejrieps/514

DOI : 10.4000/ejrieps.514

ISSN : 2105-0821

Éditeur

ELLIADD

\section{Référence électronique}

Jean-Francis Gréhaigne, "Les jeux à effectif réduit : plaisir et apprentissage », eJRIEPS [En ligne], Horssérie $N^{\circ} 2$ | 2018, mis en ligne le 01 juillet 2018, consulté le 01 août 2019. URL : http:// journals.openedition.org/ejrieps/514; DOI : 10.4000/ejrieps.514

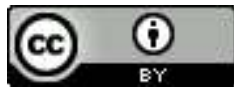

La revue eJRIEPS est mise à disposition selon les termes de la Creative Commons Attribution 4.0 International License. 


\section{Les jeux à effectif réduit : plaisir et apprentissage}

Une plage, une cour d'école, un stabilisé au bas de la tour avec deux groupes de joueurs qui s'affrontent pour la possession d'un ballon ou d'un objet qui en fait office, et voilà la magie du jeu qui s'installe. A l'origine du jeu, il y a cette liberté, cette liberté première qui en est le moteur indispensable faite de détente, de distraction et de sérieux. Pour les jeunes joueurs et les moins jeunes aussi, il est bien plus amusant de jouer avec de petites équipes sur un petit terrain avec de petits buts. Dans ces rencontres à effectifs réduits, le gain du match est recherché à l'aide de choix pertinents dans le jeu pour permettre d'accéder régulièrement à la zone de marque et de marquer. Cela peut être un simple délassement avec uniquement le plaisir de jouer, mais aussi une activité encadrée qui permet des apprentissages.

Les jeux à effectif réduit, couramment appelés jeux réduits, sont conçus pour aider les joueurs et les équipes à s'améliorer dans une variété de domaines tels que les permutations dans le jeu, l'utilisation de la périphérie, le lancement de contre-attaques, et plus encore. Les jeux réduits sont également un excellent moyen de mettre les joueurs dans des situations de jeu et d'aider à leur développement. Ces jeux sont un moyen populaire d'entraîner des joueurs de tous âges ; du débutant au niveau professionnel. Le format réduit propose aux joueurs plusieurs scénarios de type jeu dans un espace qui doit être compatible avec leurs ressources. On peut également y manipuler les règles, des restrictions pour mettre l'accent sur les différents aspects tactiques du jeu qui vont entraîner des progrès techniques. Quand on utilise des jeux réduits dans l'apprentissage, les joueurs jouent à une version modifiée de la pratique sociale de référence qui devrait leur permettre de construire des connaissances et des compétences motrices en situation. Le jeu réduit conserve les éléments importants de la complexité tactique, mais permet une exécution avec un niveau d'exigences physiques (espace réduit) et un temps plus important (densité des joueurs moins importante) qui correspondent mieux au niveau des débutants (Zerai, Gréhaigne, \& Godbout, 2011).

Cette approche permet au débutant d'enrichir son niveau de compétence actuel et son expertise se développe en termes de perception du jeu, de sens tactique, de prise de décisions et d'exécution (Kirk \& McPhail, 2002). Fait important, les jeux réduits impliquent le développement de compétences telles que la réflexion stratégique et la résolution de problèmes, deux aspects importants mais souvent sous-estimés car renvoyant aux habiletés cognitives de haut niveau (Resnick,1987). Le choix d'un jeu à effectif réduit (quatre contre quatre par exemple) limite le nombre d'informations à prélever pour le joueur novice. Dans une équipe comprenant un nombre de joueurs plus important, les informations essentielles sont noyées dans un ensemble trop vaste, et la 
perception est le plus souvent asservie à l'émotion. En jouant à trois ou quatre partenaires, il est possible, en tous cas plus facile, de percevoir les différentes alternatives fondamentales (Kawa, 1998). Les élèves sont aussi davantage impliqués dans les jeux (plus de mouvements et de situations) car comme il y a moins de joueurs sur le terrain. Les plus faibles sont sûrs de toucher la balle plus souvent ce qui garantit une plus grande attention individuelle de chacun et des progrès. Les joueurs ont aussi plus d'opportunités de marquer des buts. Le ballon change souvent d'équipe car même si les séquences de jeu deviennent un peu plus longues, elles dépassent rarement quatre ou cinq échanges de balle. Les élèves sont beaucoup plus solidaires car ils sont à la fois impliqués dans les actions d'attaque et de défense, étant ainsi plus souvent exposés à toutes sortes de situations de jeu. Dans les jeux réduits, chaque joueur ou joueuse joue tout le temps, reçoit le ballon souvent, essaie de marquer en permanence, possède une plus grande liberté de jouer et, de ce fait, prend plus de plaisir.

\section{Pourquoi les jeux réduits}

Plusieurs éléments relevant de l'expérience ou de recherches complètent ces données sur le bénéfice que l'on peut retirer des jeux réduits en comparaison avec le football à 11. En général, les joueurs touchent le ballon 5 fois plus souvent au football à $4 \times 4$ et $50 \%$ plus qu'au football à $6 \times 6$ (Gréhaigne, 2014). Ils sont trois fois plus souvent confrontés au duel pour la conquête ou la conservation du ballon en football à 4 et deux fois plus souvent qu'au football à $6 x 6$. Les buts sont marqués en moyenne toutes les minutes au football à $4 \times 4$ et toutes les quatre minutes au football à 6x6. La dimension des terrains est elle aussi très importante. Elle doit être adaptée à l'âge, aux compétences tactiques et motrices en relation avec le nombre de joueurs. Une surface comprise entre 60 et $100 \mathrm{~m}^{2}$ par joueur semble un bon compromis.

La durée des situations constitue aussi une variable essentielle : pas trop longue afin d'éviter une fatigue trop importante qui a pour conséquence une baisse évidente de l'efficience des élèves ; pas trop courte afin de laisser le temps aux élèves de s'adapter aux conditions de jeu proposées. Une durée de 6 à 8 minutes paraît réaliste pour éviter ces deux écueils (Marle \& Gréhaigne, (2009). Cette durée permet également d'effectuer un grand nombre de rotations entre les équipes et évite ainsi les temps morts sources de comportements déviants. Enfin, la taille des cibles représente des éléments incontournables des sports collectifs. En effet, elles sont porteuses de sens pour les joueurs. Il semble donc indispensable, du moins dans un premier temps, de proposer des cibles larges et / ou en grand nombre. Ensuite, quand le jeu devient plus efficient (et que l'on ne souhaite pas immobiliser un joueur dans les buts), la taille de la cible doit être réduite avec l'utilisation, par exemple, d'une haie retournée. En effet, une cible avec une faible surface favorise davantage la 
construction du jeu pour aller au but que la recherche du tir. Il peut s'agir d'une alternative intéressante sur le plan scolaire car elle évite la domination des élèves meilleurs tireurs au détriment des autres. Enfin l'utilisation de jeu réduit facilite, par sa clarté, l'apprentissage des règles du jeu et de rapports d'opposition respectueux de l'adversaire.

\section{Jeux réduits et éthique}

Ici, deux systèmes de valeurs semblent traverser et transcender les jeux sportifs collectifs à savoir «l'esprit d'équipe » et le « respect de la règle»(Gréhaigne, Meunier, \& Vigneron, 2009). Dans un jeu, l'esprit d'équipe se manifeste par la solidarité dynamique d'un nombre variable d'individus dans la préparation et l'exécution d'une action commune. La modernité a fourni à cette valeur des champs d'application nouveaux et les compétitions sportives collectives lui ont donné, sans doute, ses formes les plus achevées. L'esprit d'équipe apparaît alors non seulement dans la réalisation de la solidarité interne du groupe sportif face à un ou à des groupes sportifs rivaux, mais encore dans l'accomplissement d'une solidarité qui, partant de l'équipe, rayonne autour d'elle. Certes, cette solidarité dynamique peut comporter des excès ou des déviations se traduisant par du chauvinisme, mais la perversion d'une valeur ne doit pas nous la faire rejeter et oublier le sens de cette valeur considérée en elle-même et pour elle-même. C'est pourquoi on peut estimer que l'esprit d'équipe a trouvé et continue à trouver dans les sports collectifs un champ d'élection pour donner un plein accomplissement à une certaine forme de solidarité.

Cependant, la valeur de l'esprit d'équipe n'aurait aucune portée si n'était pas respectée une deuxième valeur: celle du franc jeu, de jouer le jeu. Celle-ci impose à tous les participants une stricte honnêteté dans leurs actions. Sous le signe de la tradition, puis sous celui de la modernité, la fin a fréquemment justifié les moyens en ce qui concerne beaucoup d'activités. Vaincre en trichant dans une épreuve sportive individuelle ou collective, ce n'est pas vaincre, car cela est contraire à la nature ludique de la pratique. Un jeu n'existe que par la règle qui l'instaure dans sa spécificité. Il y a ce qui est obligatoire, ce qui est permis et ce qui est interdit. Hors de cette normativité, il n'y a plus de jeu et par conséquent, les victoires et les défaites n'ont aucun sens. Dans les jeux réduits, on joue souvent sans arbitre et cela souligne bien que sans la conviction de départ, partagée entre tous les joueurs antagonistes, qu'il existe des règles du jeu collectif, grâce auxquelles chacun et tous peuvent gagner et / ou perdre tout en assurant leur sécurité.

La compétition peut induire une relation d'hostilité à l'adversaire quand celui-ci n'est perçu que comme personne à dominer, à abattre alors que l'idée d'émulation dans une confrontation respectueuse représente un moyen de se comparer à autrui, d'échanger avec lui et de progresser. 


\section{Utiliser les jeux réduits}

Il s'agit par exemple de s'inscrire dans le cadre d'un projet de jeu simple lié à la progression du ballon. Nous postulons que l'apprentissage du football ne peut se passer d'un détour par le jeu réduit, voire très réduit (Marle \& Gréhaigne, 2009). Nous allons nous appuyer sur les avantages du jeu en $3 \times 3$ ou 4 × 4 afin de faciliter l'acquisition de connaissances tactiques et de compétences motrices qui fondent la formation du jeune joueur.

Ce détour ne constitue en rien une réduction des exigences, mais représente au contraire une étape essentielle dans la construction des fondamentaux de l'activité. Les contenus d'enseignement seront définis en fonction des ressources principalement sollicitées en football. Les élèves apprennent à développer et à mobiliser leurs ressources en vue d'accroître leur efficience et acquérir des aspects tactico-techniques plus complexes. Les situations sollicitent l'adaptation des ressources des joueurs
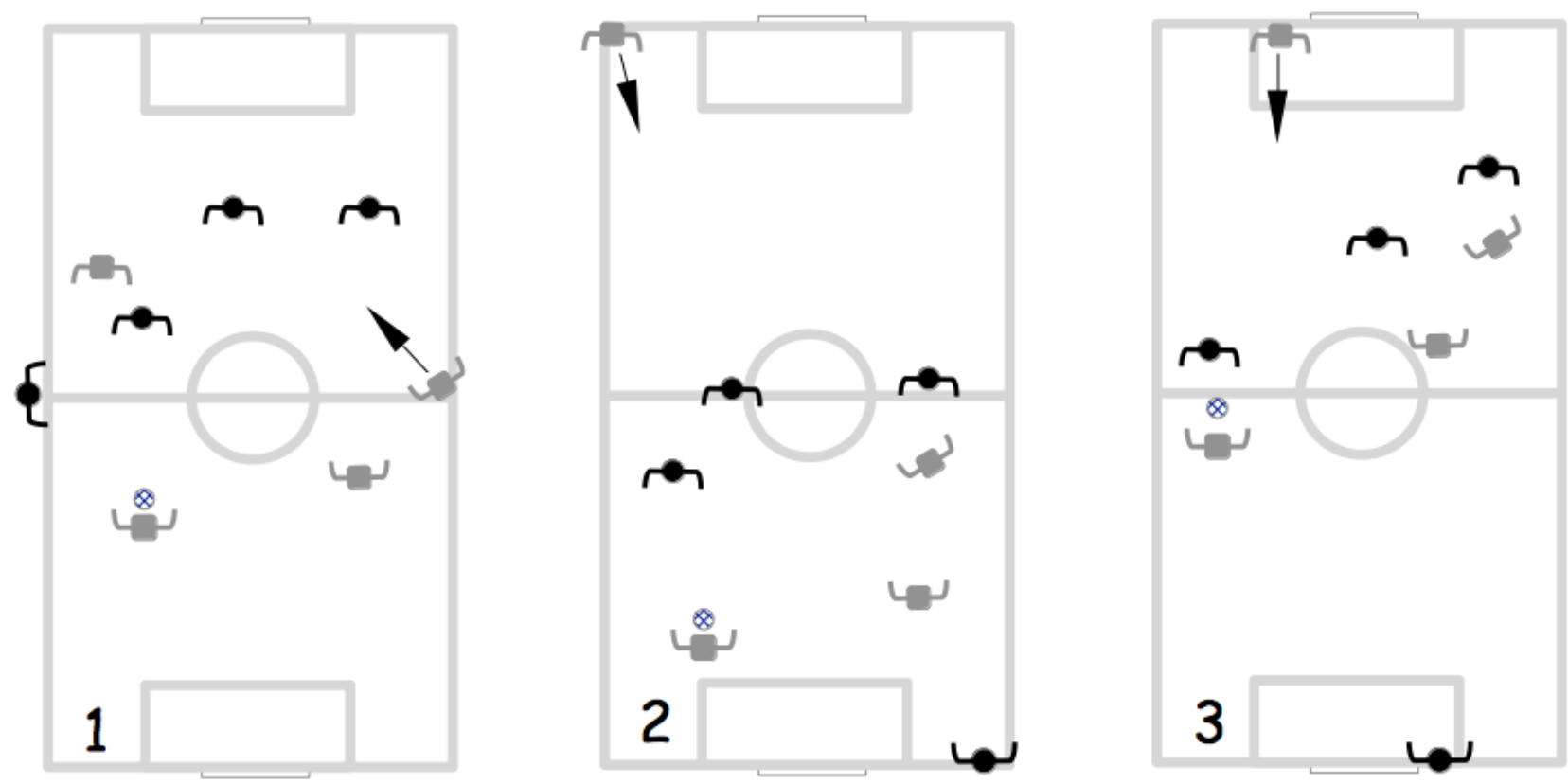

pour répondre de manière efficace à un problème précis.

Figure 1. Un joker de l'équipe attaquante entre dans le terrain à différents endroits en fonction des objectifs poursuivis.

Ici dans l'exemple (figure 1), l'organisation de la pratique repose sur un jeu en 3 x 3 plus un joker qui, selon les situations proposées, entre au milieu du terrain, au poteau de corner ou au pied du poteau de but opposé. Le jeu se déroule sans gardien avec un terrain de 40 x $20 \mathrm{~m}$ et des buts de handball, sans la règle du hors-jeu. Pour obtenir le jeu «balle en avant» et le «jeu dans l'intervalle", on place le joker sur le côté. Il rentre sur le terrain à la récupération du ballon par son équipe. Il ressort en cas de perte de balle et rejoint son poste. Le joker sur le côté rentre seulement 
quand le ballon franchit la ligne médiane. La règle d'action visée est «pour marquer, l'équipe doit amener rapidement la balle en avant de l'espace de jeu effectif ». La balle doit forcément être touchée par le joker pour qu'un but soit validé. Celui-ci a le droit, dans un premier temps, de tirer au but. Les points à observer pour l'enseignant se situent au niveau des comportements : pour les attaquants, on s'attend à des échanges de balle utilisant la largeur avec un progrès significatif lors de l'apparition du jeu long vers le joker. Dans un premier temps, ce type de jeu est un échec du fait des erreurs dans l'échange de balle entre le passeur et le réceptionneur. Rapidement ce jeu long réussi permet aux joueurs de perfectionner leurs compétences motrices. Pour la défense, on observera la qualité du recul frein et l'organisation de l'équipe en défense en infériorité numérique. Un bon indicateur de réussite est quand toutes les attaques finissent par un tir ou un but marqué (Marle, Gréhaigne, \& Caty, 2007).

Enfin, une utilisation systématique de maillots de couleurs différentes concourt à la clarté des informations perçues par les joueurs.

L'analyse des trois situations proposées montrent des aspects communs et des différences. Comme souvent dans ce type de jeu à effectif réduit, la défense est en barrage et il est très différent de rentrer au milieu du terrain ou de rentrer au fond du terrain avec un temps d'avance conséquent. Par contre pour le porteur de balle, le fait de devoir jouer avec le joker dans le cas (2) impose, fréquemment, le recours à une passe longue. Dans les rapports d'opposition, la perte de la balle à l'arrière de l'espace de jeu effectif dans tous les cas de figure entraîne immédiatement une contreattaque des anciens défenseurs avec une bonne chance de succès la défense étant hors de position. Ainsi dans ces situations, la réversibilité peut jouer à plein. On voit bien à partir de ces trois exemples l'infinie variété de configurations du jeu que peut proposer ce type de situations d'apprentissage. D'autant plus que le fait de mettre en place une défense à la poursuite au début de l'action peut changer considérablement les apports de cette confrontation. Pour un exemple d'utilisation d'un joker ou du «support player » en hockey sur glace, on peut consulter Nadeau, Gréhaigne, \& Godbout (2017).

Différentes variables peuvent être utilisées pour faire évoluer la situation comme retirer l'obligation de donner le ballon au joker, varier les modalités d'entrée du joker ou restreindre sa zone d'action. Les indicateurs de réussite vont de l'utilisation effective du joker au recours à des plans tactiques particuliers. Il est à noter que ce genre de situation d'apprentissage doit avoir une existence assez longue pour qu'elle puisse être efficace. Elle doit être présentée plusieurs fois aux élèves le temps que les processus de découverte, d'appropriation et d'apprentissage puissent s'effectuer. Dans un développement ultérieur, il est possible de recourir à la présence ou non d'un gardien tout en jouant sur les dimensions des cibles. 


\section{Jeux réduits et entraînement physique}

Par sa nature, le jeu réduit est exigeant sur le plan cardiovasculaire et musculaire (duels / changements de direction / répétition de courses, utilisation d'espaces, etc.). L'analyse de l'activité physique du joueur de football au cours d'un match montre que l'effort du joueur est essentiellement de type aérobie. Les exercices intermittents de courtes durées sont les plus consistants. Les réponses cardiovasculaires de certains jeux réduits avec ballon offrent des similitudes avec certains exercices intermittents courses de courtes durées. Il faut également proposer aux élèves des récupérations d'une durée suffisante entre les séquences de jeu.

Dans un travail préliminaire à propos de ce type de jeu, Dallancourt (1994) puis Gréhaigne \& Marchal (2002) ont mis en évidence des tendances fortes que confirment des études plus poussées menées quelques années plus tard (voir Brandes \& Elvers, 2017 pour une revue).

Dans le cas du 6 x 6, les joueurs se trouvent la majorité du temps (références) (moyenne $83 \%$ dans la zone fonctionnelle de stimulation du système aérobie, à des FC comprises entre $70 \%$ et $90 \%$ de la fréquence cardiaque maximale (FC max.), c'est-à-dire pour la plupart des élèves à des FC comprises entre 150 et 190 pulsations/minutes (de $54 \%$ à $91 \%$ du temps). Les périodes où les élèves dépassent $90 \%$ de FC max. vont de $2 \%$ à 35,5\% (en moyenne $=16 \%$ ). Les périodes où les élèves sont en-dessous de $70 \%$ de FC max. vont de $0 \%$ à $8 \%$ (moyenne $2 \%$ ) Les FC moyennes relevées vont de 157,5 à 181,5 puls/min. Elles se situent entre 75 et $86 \%$ de FC max. (moyenne à $83 \%$ de FC max. par rapport à des FC max. de 210 puls/min).

Dans le cas du 4 x 4, là encore, les joueurs testés ont des FC comprises la majorité du temps entre 150 et 190 pulsations / minute. Les données recueillies vont de 51,5\% à $99 \%$ du temps passé dans cette zone. Les périodes où les élèves dépassent $90 \%$ de FC max. sont beaucoup plus nombreuses. Les FC moyennes des élèves vont de 173,5 à 189,5 puls/min pour les élèves possédant des FC max. de l'ordre de 210 puls/min (moyenne 184 puls/min sur 31 données, c'est-à-dire 87,5 \% de FC max.). En conclusion, il semble que les deux formats de jeux réduits utilisés dans la présente étude sont efficaces pour produire des intensités de travail compatibles avec le développement de la puissance aérobie maximale. Enfin, à l'entraînement, un autre avantage de ces types de jeu est que les joueurs perçoivent de manière significative ces jeux à effectif réduit comme moins difficiles et lassants que les courses classiques, malgré des réponses cardiovasculaires quasiment identiques.

\section{Conclusion}

Quel que soit le type de société, les jeux sportifs possèdent des traits communs et continus dont les caractéristiques essentielles peuvent être énoncées ainsi : ces jeux sont libres, gratuits, sérieux, organisés, réglés (Chateau, 1946) et en cela ils s'opposent à toute pratique professionnelle. 
Dans les jeux à effectif réduit, ces caractéristiques se retrouvent car les joueurs peuvent être confrontés à leurs propres niveaux en fonction de leur d'âge et de leurs capacités pour améliorer leur performance. S'il y a trop de joueurs sur un terrain de jeu, cela engendre des effets négatifs dans les apprentissages, limite la compréhension et les progrès des joueurs dans le jeu. Les jeunes pratiquants et les moins jeunes jouent au football d'abord pour s'amuser. Ils veulent courir, toucher la balle et participer au jeu. Aussi, l'environnement dans lequel sont placés les joueurs pendant la pratique devrait viser à promouvoir tous ces désirs.

Le jeu n'est peut-être pas aussi gratuit qu'une première acception pouvait le laisser penser, car il permet une alternance fonctionnelle entre des centrations sur son propre développement et des centrations sur les relations avec les autres. Avec Wallon (1941) on parlera, alors, de processus d'autonomisation (insertion sociale) qui, dans ce cas, s'opposent aux processus d'individualisation au sein de la société (développement individuel de la personne). Dans notre époque de non solidarité et d'individualisme concurrentiel, il nous semble important de rappeler ces quelques valeurs sociales du jeu.

\section{Bibliographie}

Brandes, M., \& S. Elvers (2017). Elite Youth Soccer Players' Physiological Responses, TimeMotion Characteristics, and Game Performance in 4 vs. 4 Small-Sided Games: The Influence of Coach Feedback. The Journal of Strength \& Conditioning Research, 31(10): 2652-2658.

Chateau, J. (1946). Le jeu de l'enfant. Paris : Vrin.

Dallancourt, T. (1994). Consommation maximale d'oxygène et jeux réduits. Mémoire de Maîtrise (non publié). Université de Bourgogne.

Gréhaigne, J.-F. (Ed.). (2014). L'intelligence tactique. Des perceptions aux décisions tactiques en sports collectifs. Besançon : Presses de l'Université de Franche-Comté.

Gréhaigne, JF., \& Marchal, D. (2004). Contribution au développement du système aérobie à l'école : un exemple à partir du football. Rapport de recherche pour le pôle Nord-Est des IUFM. IUFM de Franche-Comté.

Gréhaigne, JF., \& Meunier, J.-N., \& Vigneron, C. (2007). Éthique, arbitrage, règles et sports collectifs à l'école. In J.-F. Gréhaigne (Ed.) Configurations du jeu. Débat d'idées et apprentissage du football et des sports collectifs (pp. 123-132). Besançon : Presses de l’Université de Franche-Comté. 
Kawa, A. (1998). Le football en éducation physique au collège. Poitiers : CRDP de PoitouCharentes.

Kirk, D., \& McPhail, A. (2002). Teaching games for understanding and situated learning: Rethinking the Bunker-Thorpe model. Journal of Teaching in Physical Education, 21, 177-192.

Marle, P., Gréhaigne, J.-F., Caty, D. (2007). Pour une pédagogie des sports collectifs à l'école. In J.-F. Gréhaigne, J.-F. (Ed.) Configurations du jeu, débat d'idées et apprentissage des sports collectifs (pp. 21-42). Besançon : Presses de l’Université de Franche-Comté.

Marle, P., \& Gréhaigne, J.-F. (2009). Football. www.contrepied.net

Nadeau, L., Gréhaigne, J.-F., \& Godbout, P. (2017). Developing tactical knowledge with the help of support players: An illustration in ice hockey. International Journal of Physical Education 54 (1) 22-33.

Resnick, L.B. (1987) Education and learning to think. Washington :National Academy Press.

Wallon, H. (1941). L'évolution psychologique de l'enfant. Paris : Armand Colin.

Zerai, Z., Gréhaigne, J.F., \& Godbout, P. (2011). Des signes de ressemblance dans les configurations du jeu. In J.F. Gréhaigne (Ed.) Des signes au sens. Le jeu, les indices, les postures et les apprentissages dans les sports collectifs à l'école (pp. 73-84). Besançon: Presses de l'Université de Franche-Comté. 